\title{
Effect of wing length on the prevalence of trypanosomes in Glossina morsitans morsitans in eastern Zambia
}

Cornelius Mweempwa ${ }^{1,2^{*}}$, Kalinga Chilongo ${ }^{2}$, Kyoko Hayashida ${ }^{3}$ and Boniface Namangala ${ }^{4}$

\begin{abstract}
Background: Tsetse flies (Diptera: Glossinidae) transmit trypanosomiasis (sleeping sickness in humans and nagana in livestock). Several studies have indicated that age, sex, site of capture, starvation and microbiome symbionts, among others, are important factors that influence trypanosome infection in tsetse flies. However, reasons for a higher infection rate in females than in males still largely remain unknown. Considering that tsetse species and sexes of larger body size are the most mobile and the most available to stationary baits, it was hypothesized in this study that the higher trypanosome prevalence in female than in male tsetse flies was a consequence of females being larger than males.

Methods: Black screen fly rounds and Epsilon traps were used to collect tsetse flies in eastern Zambia. Measurement of wing vein length and examination for presence of trypanosomes in the flies were carried out by microscopy. Principal component method was carried out to assess the potential of wing vein length as a predictor variable. The multilevel binary logistic regression method was applied on whole data, one-method data and one-sex data sets to evaluate the hypothesis.
\end{abstract}

Results: Data derived from a total of 2195 Glossina morsitans morsitans were evaluated (1491 males and 704 females). The wing length variable contributed the highest variance percentage (39.2\%) to the first principal component. The variable showed significant influence on prevalence of trypanosomes when the analysis was applied on the whole data set, with the log odds for the prevalence of trypanosomes significantly increasing by $0.1(P=0.032)$, per unit increase in wing length. Females had higher trypanosome prevalence rates than males, though not always significant. Furthermore, moving from females to males, wing length significantly reduced by $0.2(P<0.0001)$.

Conclusions: We conclude that wing length is an important predictor variable for trypanosome prevalence in Glossina morsitans morsitans and could partially explain the higher prevalence of trypanosomes in females than in males. However, reasonably representative population data are required for analysis - a serious challenge with the current tsetse sampling methods. Thus, analysis combining data from mobile and stationary methods that include both sexes' data could be useful to verify this hypothesis.

Keywords: Wing length, Prevalence of trypanosomes, Glossina morsitans morsitans, Tsetse sampling methods

*Correspondence: corn62mweempwa@gmail.com

${ }^{1}$ Department of Veterinary Tropical Diseases, Faculty of Veterinary

Science, University of Pretoria, Pretoria, South Africa

Full list of author information is available at the end of the article permits use, sharing, adaptation, distribution and reproduction in any medium or format, as long as you give appropriate credit to the original author(s) and the source, provide a link to the Creative Commons licence, and indicate if changes were made. The images or other third party material in this article are included in the article's Creative Commons licence, unless indicated otherwise in a credit line to the material. If material is not included in the article's Creative Commons licence and your intended use is not permitted by statutory regulation or exceeds the permitted use, you will need to obtain permission directly from the copyright holder. To view a copy of this licence, visit http://creativecommons.org/licenses/by/4.0/. The Creative Commons Public Domain Dedication waiver (http://creativeco mmons.org/publicdomain/zero/1.0/) applies to the data made available in this article, unless otherwise stated in a credit line to the data. 


\section{Background}

Tsetse flies (Diptera: Glossinidae) transmit trypanosomiasis, a neglected tropical disease (NTD) [1]. Sampling of tsetse flies, and examination for trypanosomes in them, is a prerequisite for studies on demography, biometrics, genetics, epidemiology, diagnostics and therapeutics, in relation to management of trypanosomiasis. Sampling tsetse flies involves the use of stationary and/or mobile techniques. These techniques are known to display inherent biases toward certain sections of populations, e.g. toward particular species, sex, age group and pregnancy stage [2, 3]. For example, stationary traps are known to sample more Glossina pallidipes than Glossina morsitans morsitans, more females than males and more old than young flies [2, 3]. On the other hand, mobile methods (such as "fly rounds") are known to sample more G. $m$. morsitans than G. pallidipes, more males than females and more young than old flies [2,3]. Stationary methods (trap devices) are known to sample more of the larger than the small flies [4]. In a study by Hargrove [2], age structures determined from samples of tsetse flies caught using different methods differed significantly in six out of ten pair comparisons in G. m. morsitans and in all ten pair comparisons in G. pallidipes, and the differences were attributed to increased flight activity with age. Some studies have been carried out to identify factors that influence rates of trypanosome infection in tsetse flies and these factors include age [5], sex and site of capture [6], starvation [7] and microbiome symbionts [8, 9] among others.

As species and sexes of tsetse flies of larger body size are the most mobile [10] and are also the most available to stationary baits [11], it was hypothesized in this study that the higher rate of trypanosome prevalence in female than in male tsetse flies described in G. pallidipes [12], Glossina palpalis palpalis, Glossina tachinoides and Glossina morsitans submorstans [6] was a consequence of females being larger than males on average, with the smallest females being smaller than the largest males [13]. However, in laboratory-bred G. m. morsitans, infection rates by trypanosomes of the subgenus Trypanozoon in salivary glands were shown to be higher in males than in females $[14,15]$. Considering that body size was shown to have an influence on mobility and females tend to be larger than males, this study was conducted to investigate whether body size (measured as wing vein length) had an influence on prevalence of trypanosomes in tsetse flies.

\section{Methods}

\section{Study area and sampling of tsetse flies}

The data were collected in eastern Zambia during a study on the status of tsetse populations and epidemiology of livestock trypanosomiasis in areas of varying degrees of habitat fragmentation [16]. The description of the study area, methods used to sample tsetse populations and laboratory examinations of tsetse flies were as provided in previous studies in the area $[4,17,18]$.

In brief, sampling of tsetse flies was carried out monthly for 1 year from four sites of varying levels of habitat fragmentation [18]. The degree of fragmentation increased in the order Lusandwa, Zinaka, Chisulo and Kasamanda study sites $[17,18]$. The shortest and longest distances apart between study sites were 10 and $30 \mathrm{~km}$, respectively. Tsetse density increased as degree of fragmentation reduced [17]. Black screen fly rounds [19] and Epsilon traps [20] were used to sample tsetse populations. Collected tsetse flies were subjected to (i) sorting by sex, (ii) examination for trypanosome infection by microscopy [21], (iii) measurement of wing vein length [22], (iv) dissection of females for ovarian aging [23] and (v) assessment of wing fray for aging purposes in males [24].

\section{Data analysis}

Although two tsetse species (G. m. morsitans and G. pallidipes) were collected in the study area [18], only G. $m$. morsitans data, from both sampling methods (fly rounds and Epsilon traps), were subjected to the analyses. Very few G. pallidipes were collected and not at all the sites. Few flies (both species) were caught in the Kasamanda site, and consequently the data were excluded from the study. In the analyses, data from the three sites considered (Chisulo, Lusandwa and Zinaka) were pooled.

In analysis of the influence of wing length on prevalence of trypanosomes in G. m. morsitans, the data used were from all flies examined for presence of trypanosomes that also had their right wing length measured (the measurement of the left wing was used where the right wing measurement was missing). Considering the type of data usually available under field conditions, three types of data sets that differed in inclusiveness of variables of interest were analyzed. First were wing length data from both sampling methods (i.e. fly rounds and trapspooled) and from both sexes, here referred to as the "whole data set." Second were wing length data from only one method (i.e. either fly round or trap) but with both sexes' data included, here referred to as "one-method data set," and lastly, data from one sex (i.e. either males or females) but both methods' or one method's data analyzed, herein referred to as "one-sex data set."

Because the data sets were clustered in study sites, multilevel binary logistic regression was applied taking study site as a random variable. Permutation logistic regression models on prevalence of trypanosomes were run for the different data sets. The predictor 
variables used included method, season, sex and wing vein length (herein referred to as wing length). The variable wing length was standardized prior to analysis. The Levene's test for equality of variance was applied on wing length data between males and females for respective sites before application of the Student's and Welch's $t$ test (an adaptation of the former) to compare the means. Additional analyses were carried out to gain more insight and confidence into the results. The likelihood ratio test was carried out to compare models and determine the best fit. For each data set, the less than full models were each compared with the full model.

The variable inflation factor (VIF) was calculated to check for multicollinearity among the various model variables. Predictor variables with VIF values $>5$ were considered to have multicollinearity issues, i.e. they made the model less able to explain the relationship between the response and predictor variables.

The principal component method, multiple factor analysis [25] in particular, was carried out to determine how much variance each predictor variable contributed to the first principal component as a way to tell whether a variable was a potential predictor. Variables that contributed most variance to the first principal component were considered potential predictors [26]. The sample size used in each model was checked for sufficiency for use in logistic regression analysis using methods of Bujang et al. [27] and Peduzzi et al. [28]. The R statistical software [29] was used in the analysis.

\section{Results}

\section{Numbers of flies collected and infected}

A total of 2375 G. m. morsitans had their wing vein length measured, and 2464 were dissected for trypanosome infection examination, of which 2195 (1491 males and 704 females) were used in multilevel binary regression and principal component analyses (Table 1).

\section{Wing length and prevalence of trypanosome infection}

As Fig. 1 shows, the Lusandwa site recorded the longest mean wing length in the females $(1.75 \mathrm{~mm})$ whilst
Chisulo site had the longest mean wing length in the male flies $(1.58 \mathrm{~mm})$. Unequal variance in wing lengths between males and females was observed on Lusandwa and Zinaka sites samples, $F_{(11,122)}=38.0$ and $F_{(1923)}=$ 7.8 , respectively, $P<0.0001$. The Chisulo site samples had equal wing length variance between males and females, $F_{(1305)}=0.1, P=0.7801$. The Welch's $t$ test results showed that the mean wing length was significantly longer in females than in males at Lusandwa and Zinaka, $t_{(768.3)}=25.8$ and $t_{(419.9)}=29.7$, respectively $(P<0.0001)$, with degrees of freedom shown in parentheses. No significant difference in mean wing length between females and males at Chisulo site was observed after applying the Student's $t$ test, $t_{(305)}=0.4(P=$ 0.6989). Regarding prevalence of trypanosomes, except for trap results in Zinaka (red box), female flies had higher prevalence than male flies at all study sites and sampling methods (Fig. 2). The difference in prevalence was significant between those of male and female flies caught from fly rounds in Zinaka and those of counterpart sexes at Chisulo and Lusandwa as evidenced by the non-overlapping $95 \%$ confidence intervals.

\section{Multilevel binary logistic regression analysis}

The multilevel binary logistic regression results showed that the wing length was an important predictor variable of the prevalence of trypanosomes in G. m. morsitans. However, this was only true in some permutation models $(3 / 4)$ of only the whole data set, which did not have sex as one of the predictor variables. In models that had both the wing length and sex as predictor variables (4), both variables did not show significant influence on the prevalence of trypanosomes $(P>0.360$ and 0.078 , respectively). In the permutation model where the wing length variable showed the strongest influence on prevalence of trypanosomes, i.e. the one that had the method, season and wing length as predictor variables, model 1c (Table 2), for per unit increase in wing length, the $\log$ odds for the prevalence of trypanosomes significantly increased by $0.1(P=0.032)$. The wing length variable of the model with method and wing length as the only predictor variables showed the weakest but

Table 1 Status of trypanosomes presence in Glossina morsitans morsitans in eastern Zambia

\begin{tabular}{|c|c|c|c|c|c|c|}
\hline \multirow[t]{2}{*}{ Study site } & \multicolumn{2}{|c|}{ Cold season } & \multicolumn{2}{|c|}{ Hot season } & \multicolumn{2}{|c|}{ Rainy season } \\
\hline & Negative & Positive & Negative & Positive & Negative & Positive \\
\hline Chisulo & 73 & 23 & 13 & 8 & 113 & 42 \\
\hline Lusandwa & 243 & 121 & 71 & 30 & 427 & 180 \\
\hline Zinaka & 302 & 18 & 103 & 13 & 385 & 30 \\
\hline Total & 618 & 162 & 187 & 51 & 925 & 252 \\
\hline
\end{tabular}


significant influence on prevalence of trypanosome, per unit increase in wing length; the log odds significantly increased by 0.1 ( $P=0.044-$ model results not shown).

In permutation models for the one-method data set (4), i.e. fly round data set, per unit increase in wing length, there too were increases in the log odds by 0.1 as shown in a representative model (Table 2, model 1c). However, these increases were not significant $(P>0.068)$. No logistic regression analysis was run on the trap-only data set because the sample size of 224 was lower than the minimum of 250 required for the regression analysis. The wing length variable did not show significant influence on prevalence of trypanosomes in all other permutation models of other data sets that had sufficient sample sizes for analysis.

The sex variable also showed significant influence on prevalence of trypanosomes as exemplified in permutation models without the wing length as one of the predictor variables, built from both whole data set (4/4) and one-method (fly round) data sets (2/2). Representative models are $1 b$ and $2 b$, respectively (Table 2 ). The log odds for prevalence of trypanosomes significantly reduced by $0.3(P=0.010)$ on moving from females to males in the model (1b) with the weakest but significant influence on

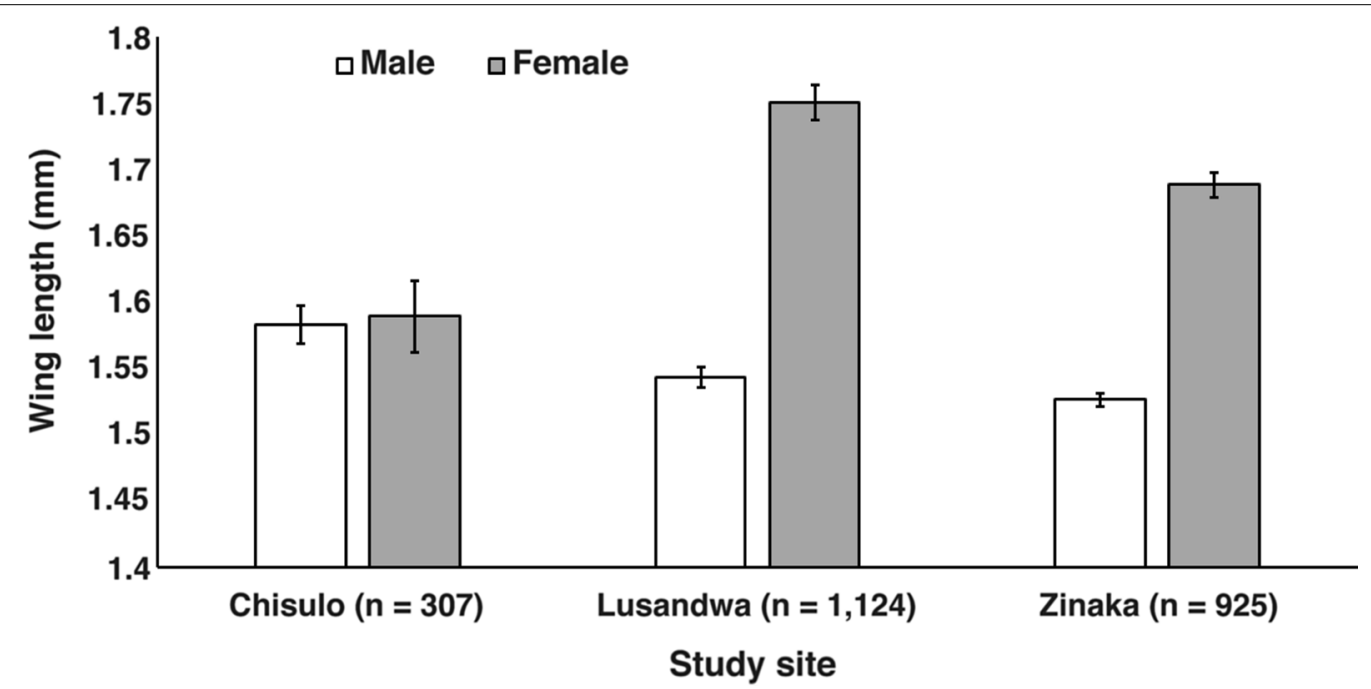

Fig. 1 Mean wing length in male and female Glossina morsitans morsitans in eastern Zambia

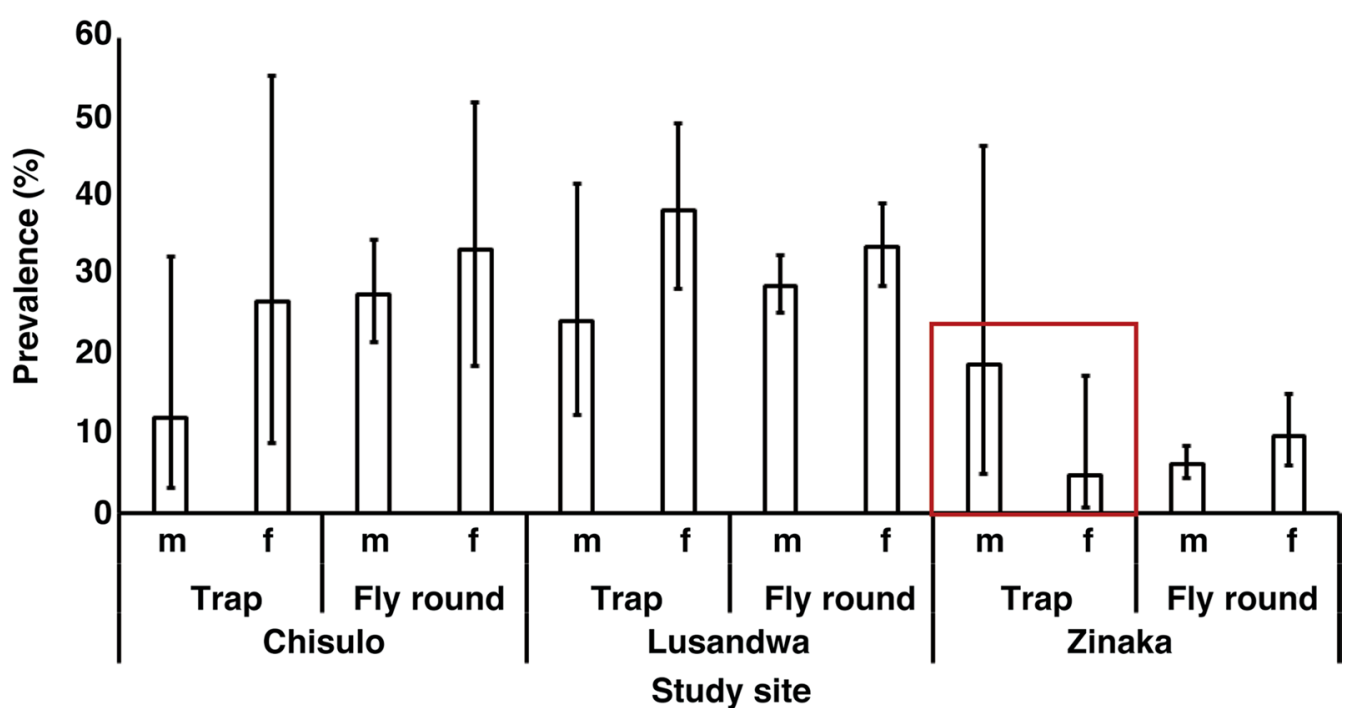

Fig. 2 Prevalence of trypanosomes in Glossina morsitans morsitans in eastern Zambia 
prevalence of trypanosomes for the whole data set and by $0.3(P=0.024)$ in the model $(2 \mathrm{~b})$ with the weakest influence on prevalence of trypanosomes for the one-method (fly round) data set. In these models without wing length as one of the predictor variables, females had significantly higher trypanosome prevalence rates than males as noted from the negative log odds values of the sex variable (Table 2). Females did not have significantly higher trypanosome prevalence rates than males in permutation models $(4 / 4$ for whole data set and $2 / 2$ for fly round data set) that included the wing length variable as one of the predictor variables as shown by representative models $1 \mathrm{a}$ and $2 \mathrm{a}$, respectively (Table 2 ).

A linear regression analysis on wing length where the sampling method, season, sex and study site were used as predictor variables for the whole data set showed that, moving from females to males, the wing length significantly reduced by $0.2(P<0.0001)$. For fly round-only and trap-only data sets, moving from females to males, the wing length significantly reduced by 0.2 and 0.1 , respectively $(P<0.0001)$.

In analysis of "females-only" data, in all permutation models of method, season, wing length and ovarian category predictor variables, only the ovarian age category variable showed significant influence on prevalence of trypanosomes $(P=0.030)$. No variable showed significant influence on prevalence in all permutation models when the "males-only" data set was analyzed using the same predictor variables as for the "females-only" data set (ovarian category variable was replaced by the wing fray variable in males-only data analysis).

Results of tests carried out to obtain further information in connection with observed regression analysis were supportive of the observations and are presented below.

\section{Likelihood ratio test}

Results of the likelihood ratio test carried out on permutation models for the whole data set showed that the sex and wing length variables together significantly improved the fit on the data set $(P=0.024)$ from the AIC $=2104.4$ for the model without the two variables to the AIC $=2100.9$ for the model with the two variables - the full model (Table 3). However, this was not the case on similar tests carried out on one

Table 2 Multilevel binary logistic regression permutation models with special reference to the wing length and the sex variables

\begin{tabular}{|c|c|c|c|c|}
\hline Logistic model & Predictor & Coefficient & $P$ value & $\mathrm{AlC}$ \\
\hline \multicolumn{5}{|l|}{1.0 whole data set } \\
\hline \multirow[t]{5}{*}{ Full model $(n=2195)$} & Trap method & -0.1 & 0.562 & 2100.9 \\
\hline & Hot season & 0.2 & 0.317 & \\
\hline & Rainy season & -0.1 & 0.673 & \\
\hline & Male sex & -0.2 & 0.090 & \\
\hline & Wing length & 0.1 & 0.370 & \\
\hline \multirow[t]{4}{*}{ Model without wing length predictor } & Trap method & 0.0 & 0.799 & 2099.7 \\
\hline & Hot season & 0.2 & 0.348 & \\
\hline & Rainy season & 0.0 & 0.701 & \\
\hline & Male sex & -0.3 & $0.010^{*}$ & \\
\hline \multirow[t]{4}{*}{ Model without sex predictor } & Trap method & -0.1 & 0.526 & 2101.8 \\
\hline & Hot season & 0.2 & 0.311 & \\
\hline & Rainy season & -0.1 & 0.631 & \\
\hline & Wing length & 0.1 & $0.032^{*}$ & \\
\hline \multicolumn{5}{|l|}{2.0 one-method data set: fly round } \\
\hline \multirow[t]{4}{*}{ Full model $(n=1971)$} & Hot season & 0.2 & 0.273 & 1863.7 \\
\hline & Rainy season & -0.1 & 0.478 & \\
\hline & Male sex & -0.2 & 0.146 & \\
\hline & Wing length & 0.1 & 0.545 & \\
\hline \multirow[t]{3}{*}{ Model without wing length predictor } & Hot season & 0.2 & 0.296 & 1862.1 \\
\hline & Rainy season & -0.1 & 0.467 & \\
\hline & Male sex & -0.3 & $0.024^{*}$ & \\
\hline \multirow[t]{3}{*}{ Model without sex variable } & Hot season & 0.2 & 0.256 & 1863.8 \\
\hline & Rainy season & -0.1 & 0.482 & \\
\hline & Wing length & 0.1 & 0.069 & \\
\hline
\end{tabular}

${ }^{*} P<0.05$ significance level 
method-only (fly round-only) data set. For the one-sex data set, i.e. females-only and males-only, no significant improvements in model fit of the data were observed by including the wing length as one of the predictor variables, $P=0.915$ and 0.152 , respectively (Table 3 ).

As for the AIC values of models for the whole data set, the model with the lowest AIC $=2095.3$ was the one where the sex variable was the only predictor variable (showed significant influence on prevalence of trypanosomes, $P=0.011$ ), followed by the one where the sex and the wing length variables were the only predictor variables AIC $=2097.0$ (showed no significant influence on prevalence of trypanosomes), $P=0.077$ and 0.580 , respectively. The model with the sex (no wing length) among the predictor variables, (model $1 \mathrm{~b}$ of Table 2) fitted the data better (AIC $=2099.7$ ) than the one with wing length (and no sex) among the predictor variables, model $1 \mathrm{c}(\mathrm{AIC}=2101.8)$.

\section{Variable inflation factor}

Results of tests for multicollinearity on full models of all data sets showed that all variable inflation factors (VIF) were $<5$ (Additional file 1: Table S1). The wing length variable from the whole data set had the highest VIF of 1.8 and the season variable from the fly round-only data set had the lowest (1.0).

\section{Principal component analysis}

Results of the principal component analysis showed that the wing length variable contributed the highest variance to the first principal component (PC1). However, it was observed that the wing length variable did so only in data sets where, except for the trap-only data set, the variable "method" was among those used in the analysis (Additional file 1: Table S2). These data sets included whole and one sex-only (females-only and males-only) data sets, $39.2,37.8$ and $33.1 \%$, respectively. In analysis of the one-method data sets in which both sexes' data were included, the wing length variable contributed the highest variance $(33.2 \%)$ to the first PC1 for the trap data set while the sex variable contributed the highest variance $[45.8 \%$-almost equal to that for the wing length variable (45.7\%)] for the fly round data set. In analyses of the one-sex data sets from one method, the site variable contributed the highest variance to the first $\mathrm{PC} 1$ for both methods data sets, except for the males-only fly round data set, where the contribution was similar to that of the wing length variable.

\section{Discussion}

This study showed that wing length was among the important factors that influenced prevalence of trypanosomes in G. m. morsitans. Detecting its importance in doing so, however, required that the data set analyzed comprised data from both sampling methods and both sexes, as shown from results of the analysis of the whole data set, model 1c (Table 2). Probably because of the sampling bias inherent in individual methods with respect to wing length [4], analysis of the one-method data set did not show the importance of wing length as an important predictor variable for prevalence of trypanosomes; the influence of the variable on prevalence seemed suppressed in the analysis. Probably, in analysis of methods pooled data, there was compensation for sampling bias shortcomings of one method by the other.

The requirement for analysis of data from the two methods to determine the importance of the wing length variable in influencing the prevalence of trypanosomes was further elucidated by results from the likelihood ratio test (Table 3 ) and the principal component analysis

Table 3 Likelihood ratio test results between the full and the less-than-full models for each data

\begin{tabular}{|c|c|c|c|c|}
\hline Data set & Test no. & Models & AIC & $\operatorname{Pr}(>$ Chisq $)$ \\
\hline \multirow[t]{4}{*}{ Whole data $(n=2195)$} & & Full model & 2100.9 & \\
\hline & 1 & No sex and wing length variables model & 2104.4 & $0.024^{*}$ \\
\hline & 2 & No wing length variable model & 2099.7 & 0.371 \\
\hline & 3 & No sex variable model & 2101.8 & 0.091 \\
\hline \multirow[t]{4}{*}{ Fly round-only data $(n=1971$} & & Full model & 1863.7 & \\
\hline & 1 & No sex and wing length variables model & 1865.1 & 0.067 \\
\hline & 2 & No wing length variable model & 1862.1 & 0.546 \\
\hline & 3 & No sex variable model & 1863.8 & 0.147 \\
\hline \multirow[t]{2}{*}{ Females-only data set $(n=704)$} & & Full model & 725.8 & \\
\hline & & No wing length variable model & 723.8 & 0.915 \\
\hline \multirow[t]{2}{*}{ Males-only data set $(n=1491)$} & & Full model & 1334.6 & \\
\hline & & No wing length variable model & 1334.7 & 0.152 \\
\hline
\end{tabular}


(Additional file 1: Table S2). The likelihood ratio test results showed the importance of the wing length variable through its contribution to model fit on the whole data set, while the principal component analysis showed it through its contribution to variance in the data pooled from both methods, thereby demonstrating its potential as a predictor variable. The principal component analysis further showed that the potential of the wing length being a predictor variable for the prevalence of trypanosomes was seen even in results of one-sex data sets, as long as the data sets included data from both sampling methods. Furthermore, results of the principal component analysis showed the suppression effect on the wing length as an important predictor variable when onemethod data were analyzed. Unlike for the whole data set, the contribution of variance by the wing length variable fell below that of the sex variable when one methodonly (fly round-only) data were analyzed. Furthermore, principal component analysis where the analyzed data set was one-sex-only data showed suppressed contribution of variance by the wing length variable (site variable had highest contribution), suggesting that the presence of data for the two sexes was also important to include in the analysis to show that the wing length variable was an important factor for prevalence of trypanosomes in G. $m$. morsitans.

In addition, the lack of multicollinearity or collinearity as noted from the low VIF values (1.0-1.8) was an indication that each variable had a unique contribution and all variables in the models together influenced the prevalence of trypanosomes without worrisome interference from each other.

Because the log odds for prevalence of trypanosomes in G. $m$. morsitans significantly increased by $0.1(P=$ $0.032)$ per unit increase in wing length and on moving from females to males, the wing length significantly reduced by $0.2(P<0.0001)$, and considering that a Glossina sex taxon with larger body size was more mobile than that of the smaller body size [10] — thus increasing the chance of acquiring an infection. Having longer wing length was associated with higher prevalence of trypanosomes, hence the higher prevalence in females than in males, thereby supporting the hypothesis. If at the age when most susceptible to infection, i.e. $24 \mathrm{~h}$ post-eclosion, the susceptibility to infection was similar between females and males [30], then at that age susceptibility was less likely to be the cause of higher prevalence in females. Since midgut-only infections formed part of the data analyzed in this study, probably the host preference and the feeding frequency differences between females and males (if any) could be a possible reason for higher prevalence of trypanosomes in females at older age. This study brought forth the wing length variable as not only an important factor that influenced the prevalence of trypanosomes in tsetse but also as an associable reason for higher prevalence in females than in males of G. $m$. morsitans.

The lack of significant difference in mean wing length between male and female flies at Chisulo could be attributed to size-dependent mortality in male flies [31-33] because of high levels of habitat fragmentation $[17,18]$.

\section{Conclusions}

We conclude that wing length is an important predictor variable for trypanosome prevalence in $G \mathrm{~m}$. morsitans and could partially explain the higher prevalence of trypanosomes in females than in males. However, reasonably representative population data are required for analysis-a serious challenge with the current tsetse sampling methods. Thus, analysis combining data from mobile and stationary sampling methods that include both sexes' data could be useful to verify this hypothesis.

\section{Abbreviations}

VIF: Variable inflation factor; PC: Principal component.

\section{Supplementary Information}

The online version contains supplementary material available at https://doi. org/10.1186/s13071-021-04907-y.

Additional file 1: Table S1. Variable inflation factors for model predictor variables. Table S2. Percentage predictor variable contribution of variance to the first three principal components (PCs) for different data sets.

\section{Acknowledgements}

This study was successfully carried out thanks to the passion of the correspondence author as a tsetse scientist shown even in his retirement time. Further thanks go to the valuable contributions of several people to whom we are very grateful: Dr. P. Sinyangwe, the Director of the Veterinary and Livestock Development Department of Zambia's Ministry of Agriculture and Cooperatives for giving permission to carry out the research, the late Prof. Peter Van den Bossche of the Institute of Tropical Medicine (ITM), Belgium, for initiating and designing methods of data collection, and field extension staff of the Veterinary and Livestock Development Department of Katete and Mambwe districts for hard work in data collection.

\section{Authors' contributions}

CM conceived the study, collected and analyzed the data and wrote the draft manuscript. KC, KH and BN revised the draft manuscript. All authors read and approved the final manuscript.

\section{Funding}

The study project was funded by Wellcome Trust, London (grant 075824/B/04/Z). Wellcome Trust was not involved in study design, collection, analysis and interpretation of data.

\section{Availability of data and materials}

The datasets during and/or analysed during the current study available from the corresponding author on reasonable request. 


\section{Declarations}

Ethics approval and consent to participate

Not applicable.

\section{Consent for publication}

Not applicable.

\section{Competing interests}

The authors declare that they have no competing interests.

\section{Author details}

${ }^{1}$ Department of Veterinary Tropical Diseases, Faculty of Veterinary Science, University of Pretoria, Pretoria, South Africa. ${ }^{2}$ Department of Veterinary Services, Tsetse and Trypanosomiasis Control Section, Ministry of Fisheries and Livestock, Lusaka, Zambia. ${ }^{3}$ Division of Collaboration and Education, International Institute for Zoonosis Control, Hokkaido University, Sapporo, Hokkaido, Japan. ${ }^{4}$ Department of Paraclinical Studies, School of Veterinary Medicine, University of Zambia, Lusaka, Zambia.

Received: 24 February 2021 Accepted: 30 July 2021

Published online: 18 August 2021

\section{References}

1. Hotez P, Molyneux DH, Fenwick A, Kumaresan J, Sachs SE, Sach JD. Control of neglected tropical diseases. N Engl J Med. 2007;357:1018-27.

2. Hargrove JW. Ovarian ages of tsetse flies (Diptera: Glossinidae) caught from mobile and stationary baits in the presence and absence of humans. Bull Entomol Res. 1991;81:43-50.

3. Warnes ML. Handbook for tsetse field staff. Estimating the distribution and abundance of tsetse flies. Zimbabwe: BMC; 1997. p. 201.

4. Mweempwa C, Mbewe NJ, De Deken R. Wing length of tsetse caught by stationary and mobile sampling methods. Acta Trop. 2020;204:105333.

5. Harley JMB. Studies on age and trypanosome infection rate in females of Glossina pallidipes aust., G. palpalis fuscipes newst. and G. brevipalpis newst. in Uganda. Bull Entomol Res. 1966;57:23-37.

6. Isaac C, Ciosi M, Hamilton A, Scullion KM, Dede P, Igbinosa IB, et al. Molecular identification of different trypanosome species and subspecies in tsetse flies of northern Nigeria. Parasit Vectors. 2016;9:301.

7. Akoda K, Van Den Abbeele J, Marcotty T, De Deken R, Sidibe I, Van Den Bossche P. Nutritional stress of adult female tsetse flies (Diptera: Glossinidae) affects the susceptibility of their offspring to trypanosomal infections. Acta Trop. 2009;111:263-7.

8. Wamwiri FN, Ndungu K, Thande PC, Thungu DK, Auma JE, Ngure RM. Infection with the secondary tsetse-endosymbiont Sodalis glossinidius (Enterobacteriales: Enterobacteriaceae) influences parasitism in Glossina pallidipes (Diptera: Glossinidae). J Insect Sci. 2014;14:272.

9. Mbewe NJ, Mweempwa C, Guya S, Wamwiri FN. Microbiome frequency and their association with trypanosome infection in male Glossina morsitans centralis of Western Zambia. Vet Parasitol. 2015;211:93-8.

10. Vale G, Hursey B, Hargrove J, Torr S, Allsopp R. The use of small plots to study populations of tsetse (Diptera, Glossinidae)_difficulties associated with population dispersal. Insect Sci Appl. 1984;5:403-10.

11. Ga V. The response of tsetse flies (Diptera, Glossinidae) to mobile and stationary baits. Bull Entomol Res. 1974;64:545-88.

12. Desta M, Beyene D, Haile S. Trypanosome infection rate of Glossina pallidipes and trypanosomosis prevalence in cattle in Amaro Special District of Southern Ethiopia. J Vet Med Anim Heal. 2013;5:164-70.

13. Glasgow JP. The Glossina community. In: Mulligan HW, editor. The African trypanosomiases. London: Gearge Allen and Unwin; 1970. p. 348-81.

14. Peacock $L$, Ferris $V$, Bailey $M$, Gibson W. The influence of sex and fly species on the development of trypanosomes in tsetse flies. PLoS Negl Trop Dis. 2012;6:e1515

15. Maudlin I, Welburn SC, Milligan P. Salivary gland infection: a sex-linked recessive character in tsetse? Acta Trop. 1991;48:9-15.

16. Mweempwa C. Status of tsetse (Glossina morsitans morsitans) populations and epidemiology of livestock trypanosomosis in areas of varying degrees of habitat fragmentation in eastern Zambia. PhD thesis. Pretoria: University of Pretoria; 2015.

17. Ducheyne E, Mweempwa C, De Pus C, Vernieuwe H, De Deken R, Hendrickx $G$, et al. The impact of habitat fragmentation on tsetse abundance on the plateau of eastern Zambia. Prev Vet Med. 2009;91:11-8.

18. Mweempwa C, Marcotty T, De Pus C, Penzhorn BL, Dicko AH, Bouyer J, et al. Impact of habitat fragmentation on tsetse populations and trypanosomosis risk in Eastern Zambia. Parasit Vectors. 2015;8:406.

19. Potts WH. A contribution to the study of numbers of tsetse-fly (Glossina morsitans Westw) by quantitative methods. S Afr J Sci. 1930;27:491-7.

20. Muzari MO, Hargrove JW. The design of target barriers for tsetse flies, Glossina spp. (Diptera: Glossinidae). Bull Entomol Res. 1996;86:579-83.

21. Lloyd LL, Johnson WB. The trypanosome infections of tsetse flies in Northern Nigeria and a method of estimation. Bull Entomol Res. $1924 ; 14: 225-7$.

22. Jackson CHN. An artificially isolated generation of tsetse flies (Diptera). Bull Entomol Res. 1946;37:291-9.

23. Saunders DS. Age determination for female tsetse flies and the age compositions of samples of Glossina pallidipes Aust., G. palpalis fuscipes Newst. and G. brevipalpis Newst. Bull Entomol Res. 1962;53:579-95.

24. Pollock JN. Training manual for tsetse control personnel, tsetse biology, systematics and distribution, vol. 1. United Nations: Food and Agriculture Organisation of the United Nations; 1982.

25. Pagès J. Analyse factorielle multiple appliquée aux variables qualitatives et aux données mixtes. Rev Stat Appl. 2002:4:5-37.

26. Kassambara A. Principal component methods in R: Practical guide. [Internet]. Statistical tools for high throughput data analysis; 2017. Available from: www.sthda.com/english/articles/31-principal-component-metho ds-in-r-practical-guide/116-mfa-multiple-factor-analysis-in-r-essentials/. Accessed 18 Jan 2021.

27. Bujang MA, Sa'at N, Tg-Abu-Bakar-Sidik TMI, Lim CJ. Sample size guidelines for logistic regression from observational studies with large population: emphasis on the accuracy between statistics and parameters based on real life clinical data. Malays J Med Sci. 2018;25:122-30.

28. Peduzzi P, Concato J, Kemper E, Holford TR, Feinstein AR. A simulation study of the number of events per variable in logistic regression analysis. J Clin Epidemiol. 1996;49:1373-9.

29. R Core Team. A Language and environment for statistical computing. 2019. R Foundation for Statistical Computing, Vienna, Austria. https:// www.R-project.org/. Accessed 12 Aug 2021.

30. Walshe DP, Lehane MJ, Haines LR. Post eclosion age predicts the prevalence of midgut trypanosome infections in Glossina. PLoS ONE. 2011;6:e26984

31. Glasgow JP. Selection for size in tsetse flies. J Anim Ecol. 1961;30:87-94.

32. Phelps RJ, Clark PY. Seasonal elimination of some size classes in males of Glossina morsitans morsitans Westwood (Diptera, Glossinidae). Bull Entomol Res. 1974:64:313-24.

33. Dransfield RD, Brightwell R, Kiilu J, Chaudhury MF, Adabie D. Size and mortality rates of Glossina pallidipes in the semi-arid zone of south-western Kenya. Med Vet Entomol. 1989;3:83-95.

\section{Publisher's Note}

Springer Nature remains neutral with regard to jurisdictional claims in published maps and institutional affiliations.

Ready to submit your research? Choose BMC and benefit from:

- fast, convenient online submission

- thorough peer review by experienced researchers in your field

- rapid publication on acceptance

- support for research data, including large and complex data types

- gold Open Access which fosters wider collaboration and increased citations

- maximum visibility for your research: over $100 \mathrm{M}$ website views per year

At BMC, research is always in progress.

Learn more biomedcentral.com/submissions 